\title{
Gestational Weight Gain: A New Factor Influencing Pregnancy Outcomes in Women with Inflammatory Bowel Disease
}

\author{
Sonia Friedman ${ }^{1}$
}

Published online: 2 June 2017

(c) Springer Science+Business Media New York 2017

Crohn's disease (CD) and ulcerative colitis (UC) are associated with adverse pregnancy outcomes, including preterm birth, small for gestational age, congenital anomalies, and stillbirths [1]. Increased disease activity during pregnancy, which is particularly worrisome, is linked to preterm birth [2]. Multiple studies of immunosuppressive medication use during pregnancy have reported that thiopurines and biologic therapies have no impact on birth outcomes [3, 4]. Nevertheless, is it only increased disease activity that physicians and patients need to be wary of? Or are there other pregnancy factors associated with adverse outcomes? Women with inflammatory bowel disease (IBD) often suffer from weight loss and malnutrition, both of which may impact a potential pregnancy. In this issue of Digestive Disease and Sciences, Bengtson et al. [5] investigated the effect of gestational weight gain (GWG) on birth outcomes using the large US Pregnancy in Inflammatory Bowel Disease and Neonatal Outcomes (PIANO) cohort of women with IBD during pregnancy. The PIANO cohort consists of 559 and 363 pregnant mothers with CD and UC, respectively, enrolled between 2006 and 2014. The authors used the Institute of Medicine guidelines for GWG according to pre-pregnancy body mass index. The problematic part of this paper was the measurement of disease activity. In the PIANO registry, measurements of the Harvey-Bradshaw Index for CD and

Sonia Friedman

sfriedman1@bwh.harvard.edu

1 Center for Crohn's and Colitis, Brigham and Women's Hospital, Harvard Medical School, 850 Boylston Street, Chestnut Hill, MA 02467, USA
Simple Clinical Colitis Index for UC were performed at each trimester. These scales, however, have not been validated for pregnant subjects; furthermore, the authors do not report corroborating fecal calprotectin concentrations. Although disease activity was also measured by the number of hospitalizations and intensification of medical therapy and/or surgery, these results were not reported.

The authors report that women with $\mathrm{CD}$ and UC with inadequate GWG had a 2.5-fold increased risk of preterm birth that was statistically significant. Furthermore, women with CD and not UC with inadequate GWG had an increased risk of intrauterine growth restriction and a trend for small for gestational age babies. The authors naturally investigated whether disease activity was the reason for poor weight gain and/or preterm birth. Although increased disease activity was associated with inadequate GWG, in mothers with disease activity, inadequate GWG was not associated with preterm birth. Conversely, in mothers without disease activity, inadequate GWG was associated with preterm birth, implying that factors other than disease activity are responsible for preterm birth in pregnant women with IBD. Since it is very difficult to measure disease activity during pregnancy, even in a prospective cohort study like PIANO, measurements were likely not completely accurate. Perhaps, if only women with moderate-severe disease activity were examined, an association would be more evident.

Since IBD affects women during their reproductive years, issues regarding pregnancy are vital to patient care. This study is very important for IBD patients considering a pregnancy because it highlights a new and important risk factor for preterm birth. Although the cause of inadequate GWG in this study is unclear, treatment of pregnant patients with IBD should focus on improving nutritional intake and maintaining disease quiescence. 


\section{Compliance with ethical standards}

Conflict of interest Dr. Friedman was a site PI at Brigham and Women's Hospital for the PIANO study. Her site enrolled 19 patients and closed in December 2015.

\section{References}

1. O'Toole A, Nwanne O, Tomlinson T. Inflammatory bowel disease increases risk of adverse pregnancy outcomes: a meta-analysis. Dig Dis Sci. 2015;60:2750-2761.

2. Nørgård B, Hundborg HH, Jacobsen BA, Nielsen GL, Fonager K. Therapeutic drug use in women with Crohn's disease and birth outcomes: a Danish nationwide cohort study. Am J Gastroenterol. 2007;102:1947-1954.

3. Mahadevan U, Sandborn WJ, Li DK, Hakimian S, Kane S, Corley DA. Pregnancy outcomes in women with inflammatory bowel disease: a large community-based study from Northern California. Gastroenterology. 2007;133:1106-1112.

4. Mahadevan U, McConnell RA, Chambers CD. Drug safety and risk of adverse outcomes for pregnant patients with inflammatory bowel disease. Gastroenterology. 2017;152:451-462.

5. Bengtson MB, Martin CF, Aamodt G, Vatn MH, Mahadevan U. Inadequate gestational weight gain predicts adverse pregnancy outcomes in mothers with inflammatory bowel disease: results from a prospective US pregnancy cohort. Dig Dis Sci. 2017. doi:10.1007/s10620-017-4547-5. 